\title{
Foreword: special issue on structure transformation
}

\author{
UGO MONTANAR I
}

\author{
Dipartimento di Informatica, Università di Pisa, \\ Pisa, Italy \\ Email: ugo@di.unipi.it
}

Received 3 February 2012

This special issue of Mathematical Structures in Computer Science is dedicated to structure transformation and contains contributions to two main research areas: extending the constructions that have been developed over nearly forty years for graph transformations to more general structures; and employing these transformations to model the operational and abstract semantics of distributed and mobile systems.

A convenient starting point for understanding the motivation and content of this special issue is the Dagstuhl Seminar 04241, held between 6 and 11 June in 2004, which was organised by Barbara König, Philippa Gardner and myself, though I was unfortunately unable to attend it because of unavoidable family reasons. The subject of the seminar was Graph transformations and process algebras for modelling distributed and mobile systems, with a focus on comparing them in terms of operational and observational semantics, concurrency, compositionality and spatial structure.

Particular attention was paid to the approach by Leifer and Milner to reactive systems and by Ehrig and König to borrowed contexts. In both cases, the idea was to start with a reduction operational semantics defined by a set of ground term rewriting rules in the case of reactive systems and double pushout (DPO) graph diagrams in the case of borrowed contexts. If the state of the system is such that it must be contextualised for matching the rule/diagram, the transition is still derivable, but it must by labelled by the required context. Furthermore, if, in the case of Leifer and Milner, a relative pushout (RPO) diagram exists, only certain minimal contexts are needed, and the resulting bisimilarity is a congruence with respect to contextualisation. In this way, reduction systems are transformed into labelled transition systems, where equivalences and congruences based on bisimilarity and modal logics can be defined. This result is particularly valuable for DPO-based graph transformations, where observational semantics had rarely been applied.

A limiting factor for Leifer and Milner's approach is that, due to state symmetries, the RPO does not exist in several important cases. However, as shown by Sassone and Sobociński, in the 2-categorical setting, the corresponding universal construction, called the grupoid relative pushout (GRPO), does exist under weaker conditions.

Much attention in the Dagstuhl seminar was paid to adhesive categories, which had been introduced by Lack and Sobocinski and were suggested as generalisations of the category of graphs, the aim being to extend well-know categorical graph transformation results to adhesive categories, or related categories, as previously attempted by Ehrig, Parisi-Presicce and others with high-level replacement systems (HLRS). 
In addition, adhesive categories were immediately welcomed as a bridge between the Leifer-Milner and Ehrig-König approaches. In fact, on the one hand, cospan bicategories on an adhesive category possess GRPOs, while, on the other hand, the borrowed context construction also works in every adhesive category. As a consequence, it becomes more convenient to model distributed process description languages using structure transformation thanks to the possibility of easily deriving an abstract compositional semantics.

After almost eight years, this Special Issue presents an up-to-date, indeed mature, view of the research triggered by the seminar. In particular, the generalisation of graph constructions to variants of adhesive categories has proved to be quite successful.

The paper Processes and unfoldings: concurrent computations in adhesive categories by Paolo Baldan, Andrea Corradini, Tobias Heindel, Barbara König and Paweł Sobociński generalises the notion of a non-sequential process and the unfolding construction, which are already known in the literature for concrete formalisms like Petri nets and graph grammars, to the abstract setting of single-pushout rewriting in adhesive categories. The main results show that deterministic, non-sequential processes are in one-to-one correspondence with switch-equivalent classes of derivations of the given system, and that the unfolding construction can be characterised as a coreflection, that is, the unfolding functor is the right adjoint to the embedding of the category of occurrence grammars into the category of grammars. As the unfolding represents potentially infinite computations, the unfolding construction is defined for adhesive categories with 'well-behaved' colimits of $\omega$-chains of monos. Compared to previous work on the unfolding of Petri nets and graph grammars, the results apply to wider classes of systems thanks to the use of a refined notion of grammar morphism.

Finitary $\mathcal{M}$-adhesive categories are $\mathcal{M}$-adhesive categories that only have finite objects, where $\mathcal{M}$-adhesive categories are a slight generalisation of weak adhesive high-level replacement (HLR) categories. An object is said to be finite if it has a finite number of $\mathcal{M}$-sub-objects. The paper Finitary $\mathcal{M}$-adhesive categories by Karsten Gabriel, Benjamin Braatz, Hartmut Ehrig and Ulrike Golas shows that not only do finitary $\mathcal{M}$-adhesive categories have all the well-known HLR properties of weak adhesive HLR categories, which are already valid for $\mathcal{M}$-adhesive categories, but also all the additional HLR requirements needed to prove the classical results. Moreover, the finitary restriction of each $\mathcal{M}$-adhesive category is a finitary $\mathcal{M}$-adhesive category, and finitarity is preserved under functor and comma category constructions based on $\mathcal{M}$-adhesive categories. Finally, the paper contains a discussion on how some of the results may be extended to non- $\mathcal{M}$ adhesive categories.

The borrowed context (BC) mechanism allows for the effective construction of a labelled transition system (LTS) that has graphs as states and spans of graphs as labels, and such that the associated bisimilarity is a congruence. The paper RPO semantics for mobile ambients by Filippo Bonchi, Fabio Gadducci and Giacoma Valentina Monreale offers a test of the applicability of BCs by considering a suitable instance of the technique and, choosing Mobile Ambients (MAs) as a testbed, focussing on the synthesis of LTSs for process calculi that do not have a labelled semantics yet. The proposal is based on a graphical encoding: a process is mapped into a graph equipped with interfaces such that 
the denotation is fully abstract with respect to the standard structural congruence. Since graphs with interfaces are also amenable to the synthesis mechanism based on BCs, an LTS for the original MA calculus is obtained. As a further refinement, a finite set of SOS rules for the distilled LTS is also derived.

Amalgamation is a well-known concept for graph transformations and can be used to model synchronised parallelism of rules with shared subrules and corresponding transformations. The paper Multi-amalgamation in $\mathcal{M}$-adhesive categories by Ulrike Golas, Annegret Habel and Hartmut Ehrig presents the theory of amalgamation for $\mathcal{M}$-adhesive categories for a bundle of rules with (nested) application conditions. The two main results are the Complement Rule Theorem, showing how to construct a minimal complement rule for each subrule, and the Multi-Amalgamation Theorem, which generalises the wellknown Parallelism and Amalgamation Theorems to the case of multiple synchronised parallelism. In order to apply the largest amalgamated rule, maximal matchings are used, and these are computed according to the actual instance graph. The constructions are illustrated by a small but meaningful running example, while a more complex case study concerning the firing semantics of Petri nets is presented as an introductory example and to provide motivation.

Nested application conditions are important for several application domains and generalise the well-known negative application conditions. The paper $\mathcal{M}$-adhesive transformation systems with nested application conditions by Hartmut Ehrig, Ulrike Golas, Annegret Habel, Leen Lambers and Fernando Orejas presents Local Church-Rosser, Parallelism, Concurrency and Amalgamation Theorems for rules with nested application conditions in the framework of $\mathcal{M}$-adhesive categories. Most of the proofs are based on the corresponding statements for rules without application conditions and two Shift Lemmas saying that nested application conditions can be shifted over morphisms and rules.

A range of specific termination criteria is available when specifying model transformations by graph transformation, for which termination is in general undecidable. Unfortunately, and particularly in the case of large and heterogeneous specifications, it is often impossible to use a single termination criterion. The paper Combining termination proofs in model transformation systems by Dénes Bisztray and Reiko Heckel proposes an approach that applies different criteria to suitable subsets of rules such that termination can be shown locally using the most suitable technique for each subset. Global termination follows if certain causal dependencies between the rules in different subsets are acyclic. The theory is developed at the level of typed attributed graphs, and is motivated and illustrated by a case study translating UML activity diagrams to CSP. Finitary $\mathcal{M}$-adhesive categories, as presented in the paper by Gabriel, Braatz, Ehrig and Golas, are discussed as a possible generalisation of the theory.

The paper Formal analysis of model transformations based on triple graph grammars by Frank Hermann, Hartmut Ehrig, Ulrike Golas and Fernando Orejas is concerned with bidirectional model transformations, which play a central role in model driven software engineering. The main focus is on analysing and ensuring important properties of such transformations based on triple graph grammars (TGGs), which are a well-established concept for the specification and execution of bidirectional model transformations. The 
paper shows that the crucial properties of syntactical correctness and completeness are ensured for the concept of TGG model transformations presented in the paper. The paper also presents semi-automated analysis and verification techniques related to (strong) functional behaviour and (complete) information preservation of the derived forward and backward transformations. To this end, a new kind of operational rule is introduced, and existing results for the analysis of local confluence known for typed attributed graph transformation systems are adapted.

The paper Analysis of permutation equivalence in $\mathcal{M}$-adhesive transformation systems with negative application conditions by Frank Hermann, Andrea Corradini and Hartmut Ehrig is concerned with the interleaving semantics of $\mathcal{M}$-adhesive transformation systems, where transformation rules can be equipped with negative application conditions (NACs), which are often used for applications. For such systems, the paper proposes an original equivalence on transformation sequences, called permutation equivalence, which is coarser than the classical switch equivalence. The paper presents a general construction of deterministic processes for $\mathcal{M}$-adhesive transformation systems with NACs based on sub-object transformation systems. As a main result, this construction is shown to be compatible with the notion of permutation equivalence, which means that the process obtained from a transformation sequence identifies its equivalence class of permutationequivalent transformation sequences. The paper also discusses how the analysis of a process of a transformation sequence can be reduced to the analysis of the reachability graph of a suitably generated place/transition Petri net.

Ugo Montanari

Guest editor of the special issue. 